ORIGINAL ARTICLE

\title{
Ets-1 mediates upregulation of Mcl-1 downstream of XBP-1 in human melanoma cells upon ER stress
}

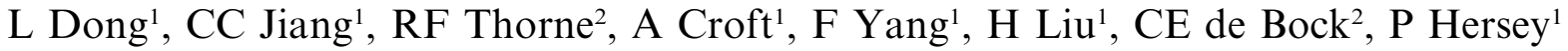 \\ and XD Zhang ${ }^{1}$ \\ ${ }^{1}$ Immunology and Oncology Unit, Calvary Mater Newcastle Hospital, Newcastle, New South Wales, Australia and \\ ${ }^{2}$ Cancer Research Unit, School of Biomedical Sciences, University of Newcastle, Newcastle, New South Wales, Australia
}

\begin{abstract}
Past studies have shown that upregulation of the antiapoptotic Bcl-2 family protein Mcl-1 is a major adaptive mechanism of melanoma cells to endoplasmic reticulum (ER) stress, and has an important role in resistance of the cells to apoptosis. In this study, we show that the increase in transcription of Mcl-1 in melanoma cells triggered by pharmacological ER stress inducers is mediated by the transcription factor Ets-1. By incremental deletion analysis of the Mcl-1 promoter, we identified a DNA fragment containing an Ets-1 binding site that is transcriptionally responsive to ER stress. Mutations in the Ets-1 binding site or knockdown of Ets-1 inhibited the increase in Mcl-1, indicating that Ets-1 has a critical role in transcriptional upregulation of Mcl-1. Similar to Mcl1, Ets-1 was transcriptionally upregulated by ER stress. This was mediated by the IRE1 $\alpha /$ XBP-1 branch of the unfolded protein response, as upregulation of Ets-1 was inhibited in melanoma cell lines deficient in IRE1 $\alpha$ or XBP-1 established by short hairpin RNA knockdown. Activation of the PI3k/Akt pathway downstream of XBP-1 was also involved, in that inhibition of the pathway blocked upregulation of Ets-1. Inhibition of Ets-1 enhanced ER stress-induced apoptosis in melanoma cell lines and in fresh melanoma isolates, recapitulating the effect of inhibition of Mcl-1. These results reveal a key mechanism by which Mcl-1 is transcriptionally upregulated in melanoma cells by ER stress, and identify Ets-1 as a potential target for inhibition to sensitize melanoma cells to apoptosis.
\end{abstract}

Oncogene (2011) 30, 3716-3726; doi:10.1038/onc.2011.87; published online 21 March 2011

Keywords: melanoma; Mcl-1; XBP-1; Ets-1; endoplasmic reticulum stress

Correspondence: Dr XD Zhang or Dr P Hersey, Immunology and Oncology Unit, Calvary Mater Newcastle Hospital, Room 443, David Maddison Clinical Sciences Building, Cnr. King \& Watt Streets, Newcastle, New South Wales 2300, Australia.

E-mails: Xu.Zhang@newcastle.edu.au or

Peter.Hersey@newcastle.edu.au

Received 5 January 2011; revised 13 February 2011; accepted 16 February 2011; published online 21 March 2011

\section{Introduction}

Melanoma continues to increase in incidence in many parts of the world, but there is currently no curative treatment once the disease has spread beyond the primary site. This is largely due to resistance of melanoma cells to induction of apoptosis by available chemotherapeutic drugs and biological reagents (Soengas and Lowe, 2003; Hersey et al., 2006). The anti-apoptotic Bcl-2 family protein Mcl-1 is of particular importance in resistance of melanoma to treatment, in that its expression increases with melanoma progression and is associated with poorer prognosis (Cory and Adams, 2002; Mhaidat et al., 2007; Wang et al., 2007; Zhuang et al., 2007; Jiang et al., 2009a). As a protein with a rapid turn-over rate, Mcl-1 expression is frequently regulated by post-translational mechanisms (Zhong et al., 2005; Warr and Shore, 2008; Schwickart et al., 2010). Nevertheless, an increase in the Mcl-1 protein levels often correlates with an increase in its mRNA levels, mostly due to enhanced transcription (Iglesias-Serret et al., 2003; Warr and Shore, 2008). Several transcription factors including HIF $1 \alpha$ and Stat 3 are known to regulate $\mathrm{Mcl}-1$ expression in various cell types (Epling-Burnette et al., 2001; Iglesias-Serret et al., 2003; Piret et al., 2005; Isomoto et al., 2005; Wang et al., 2003).

A number of cellular stress conditions, such as nutrient deprivation and alterations in glycosylation status, lead to accumulation of unfolded and/or misfolded proteins in the endoplasmic reticulum (ER) lumen and cause ER stress (Harding et al., 2002; Zhang and Kaufman, 2004; Schroder and Kaufman, 2005). The ER responds by activation of a range of signaling pathways that are collectively termed the ER stress response or the unfolded protein response (UPR) (Harding et al., 2002; Zhang and Kaufman, 2004; Schroder and Kaufman, 2005), which is fundamentally a cyto-protective response, but excessive or prolonged UPR can lead to apoptotic cell death. However, we have found that melanoma cells have largely adapted to ER stress and are resistant to ER stress-induced apoptosis (Hersey and Zhang, 2008; Jiang et al., 2008). In addition, we have shown that upregulation of Mcl-1 is a major adaptive mechanism (Hersey and Zhang, 2008; Jiang et al., 2008). Although transcriptional regulation 
was found to be involved in UPR-mediated upregulation of Mcl-1 (Jiang et al., 2008), the precise mechanism(s) responsible remains to be defined.

E26 transformation-specific sequence (Ets)-1 is a member of the Ets family of transcription factors that have roles in many biologic processes such as cell growth and survival (Dittmer, 2003; Hahne et al., 2008). Increased Ets-1 expression has been reported to correlate with grade of malignancy and poorer prognosis in a number of cancers (Davidson et al., 2001; Span et al., 2002). In melanoma, high levels of Ets-1 are often associated with progression of the disease (Poser and Bosserhoff, 2004; Rothhammer et al., 2004; Torlakovic et al., 2004). The role of Ets-1 in regulation of apoptosis seems to be cell type-dependent, as it protects against apoptosis in some cell types, but induces apoptosis in others (Bories et al., 1995; Sato et al., 2001; Teruyama et al., 2001; Dittmer, 2003). Although the mechanisms involved remain to be better understood, it is known that Ets-1 can regulate the expression of the BH3-only protein Bid in endothelial cells and the expression of Bcl-2 in colon cancer cells (Li et al., 2000; Sato et al., 2001).

We have examined the mechanism responsible for the increase in Mcl-1 at the transcriptional level in melanoma cells upon pharmacological induction of ER stress. We show in this report that transcriptional upregulation of $\mathrm{Mcl}-1$ by ER stress is mediated by Ets-1 that is also upregulated by the IRE1 $\alpha / \mathrm{XBP}-1$ branch of the UPR. Moreover, we demonstrate that activation of the PI3k/Akt pathway mediated by XBP-1 is involved in upregulation of Ets-1, and that inhibition of Ets-1 recapitulates, at least in part, the effect of inhibition of Mcl-1 on sensitivity of melanoma cells to ER stress-induced apoptosis.

\section{Results}

The region between -205 and -175 of the $\mathrm{Mcl}-1$ promoter is essential for transcriptional upregulation of Mcl-1 in melanoma cells under ER stress

Past studies have shown that a transcriptional increase is involved in upregulation of Mcl-1 in melanoma cells upon ER stress (Jiang et al., 2008). To determine the active region of the Mcl-1 promoter responsible for this, a luciferase reporter containing sequences between -1300 and +10 (pGL3-Mcl-1-1300/ + 10) (numbers relative to the transcription start site) was constructed and transiently transfected into Mel-CV and MM200 cells (Figure 1a). This region encompasses a number of predicted binding sites for various transcription factors including NF- $\kappa \mathrm{B}$, Stat3, Ets, CREBP and Sp1 (Akgul et al., 2000). Treatment with Tunicamycin (TM) that induces ER stress by inhibition of glycosylation, resulted in significant increases in transcriptional activity of the reporter in both cell lines $(P<0.05$, two-tailed Student's $t$-test) (Figure 1b) (Elbein, 1987). In contrast, exposure to TM did not cause any increase in the transcriptional activity of the luciferase reporter containing the $-2600 /$ -1200 upstream region of the Mcl-1 promoter (Supplementary Figure S1). Induction of ER stress by TM was corroborated by the increased levels of GRP78, an indicator of activation of the UPR (Supplementary Figure S2) (Jiang et al., 2008).

To further map the site within the $-1300 /+10$ region responsible for the TM-induced increase in Mcl-1 transcription, a series of incremental deletion reporter constructs contained in this region were analyzed (Figure 1a). As shown in Figure 1b, TM increased transcriptional activity in all the constructs except for pGL3-Mcl-175/+10 and pGL3-Mcl-1-65/+10.

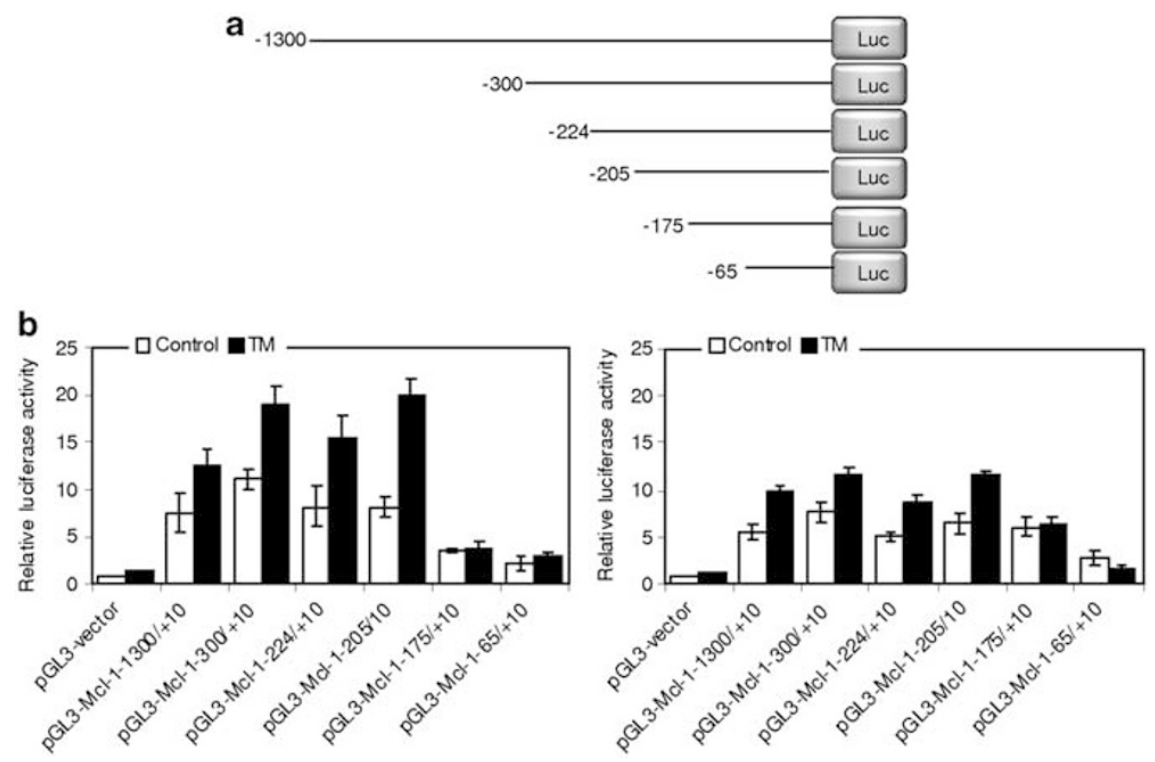

Figure 1 The $-205 /-175$ region of the Mcl-1 promoter is essential for transcriptional upregulation of Mcl-1 in melanoma cells under ER stress. (a) A schematic illustration of construction of the luciferase reporter constructs. (b) Mel-CV (left) and MM200 (right) cells were transiently transfected with indicated pGL3-basic based reporter constructs. After $24 \mathrm{~h}$, cells were treated with TM $(3 \mu \mathrm{M})$ for a further $16 \mathrm{~h}$ followed by measurement of the luciferase activity. Bars, s.e. $(n=3)$. 
The shortest fragment that was transcriptionally responsive to TM was pGL3-Mcl-1-205/+10. Therefore, the region between -205 and -175 is required for transcriptional upregulation of Mcl-1 in melanoma cells by ER stress. This was confirmed in experiments with another ER stress inducer thapsigargin (TG) (Supplementary Figure S3) (Sagara and Inesi, 1991).

\section{An Ets binding region located between -205 and -175} of the $\mathrm{Mcl}-1$ promoter is transcriptionally responsive to ER stress

The $-205 /-175$ fragment of the Mcl-1 promoter contains a consensus binding region of the transcription factor c-Rel (Figure 2a) (Akgul et al., 2000), which is known to participate in regulation of cell survival (Chen et al., 2000). We therefore tested if this region has a role in activation of the Mcl-1 promoter by ER stress. Mutation of the region abolished the transcriptional activity of the luciferase reporter after treatment with TM (Figures 2a and b). However, there was no association between c-Rel and the segment spanning from -205 to +10 of the Mcl-1 promoter as shown in chromatin immunoprecipitation (ChIP) assays (Figure 2c), suggesting that transcriptional activation of the $-205 /-175$ fragment by ER stress is not mediated by c-Rel.
Intriguingly, within the c-Rel consensus motif is also a predicted binding site for Ets transcription factors (Akgul et al., 2000). We therefore examined if Ets-1 and -2 are involved in activation of the region. ChIP assays demonstrated that Ets-1, but not Ets-2, bound to the $-205 /+10$ segment, which was enhanced by treatment with TM (Figure 2c). Binding of Ets-1 to the region was also confirmed in electrophoretic mobility shift assay (EMSA) (Figure 2d). The nuclear protein complexed with the labeled probe (the segment from -205 to -175 ) was diminished with the addition of an antibody against Ets-1, but not an antibody against c-Rel or Ets-2. A supershift in the presence of an anti-Ets1 antibody was not observed in the EMSA assay, presumably due to steric interference between the antibody and oligonucleotide binding, which prevented formation of the antibody-DNA-protein super-complex, as reported previously (Myers et al., 2005; Zhang et al., 2010). Nevertheless, these results suggest that binding of Ets-1 to the $-205 /-175$ region may be responsible for activation of the Mcl-1 promoter in melanoma cells under ER stress.

\section{Ets-1 is responsible for transcriptional upregulation of $\mathrm{Mcl}-1$ in melanoma cells upon ER stress}

As shown in Figure 3a, TM induced a rapid increase in Ets-1 along with Mcl-1 at the protein level. Similarly,

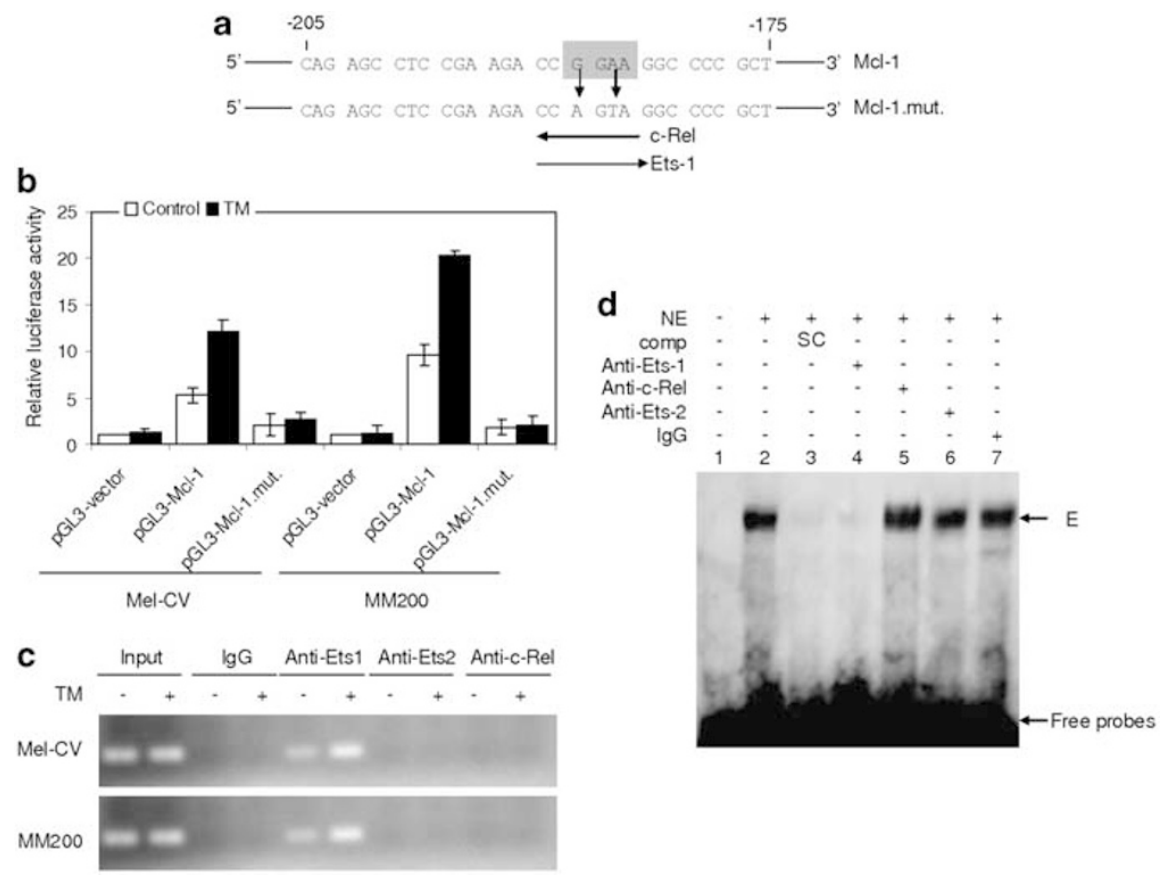

Figure 2 An Ets binding region between -205 and -175 of the Mcl-1 promoter is transcriptionally responsive to ER stress. (a) A schematic illustration of a potential c-Rel and Ets-1 binding motif located within the $-250 /-175$ region of the Mcl-1 promoter. The Ets consensus binding motif is boxed in highlighted in gray. Experimental mutagenesis of the region is also depicted. (b) Mel-CV and MM200 cells were transiently transfected with the pGL3-basic-based reporter constructs, pGL3-vector, pGL3-Mcl-1-205/ + 10 (pGL3-Mcl-1), and a reporter with the c-Rel binding region mutated as shown in (a) (pGL3-Mcl-1-mut), respectively. After $24 \mathrm{~h}$, cells were treated with TM $(3 \mu \mathrm{M})$ for a further $16 \mathrm{~h}$ followed by measurement of the luciferase activity. (c) Formaldehyde-cross-linked

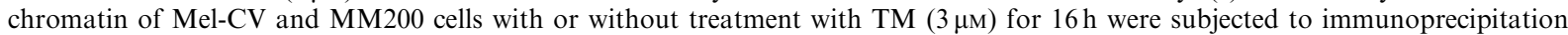
with antibodies against Ets-1, Ets-2 and c-Rel, respectively. The precipitates were subjected to PCR amplification using primers for the $-205 /+10$ region of the Mcl-1 promoter. (d) Nuclear extracts of MM200 cells with treatment with TM ( $3 \mu \mathrm{M})$ for $16 \mathrm{~h}$ were subjected to super shift assay. Comp: competitor; NE, nuclear extracts; SC, specific competitor. E, the complex formed in the presence of nuclear extracts with labeled oligonucleaotides. Bars, s.e. $(n=3)$. 

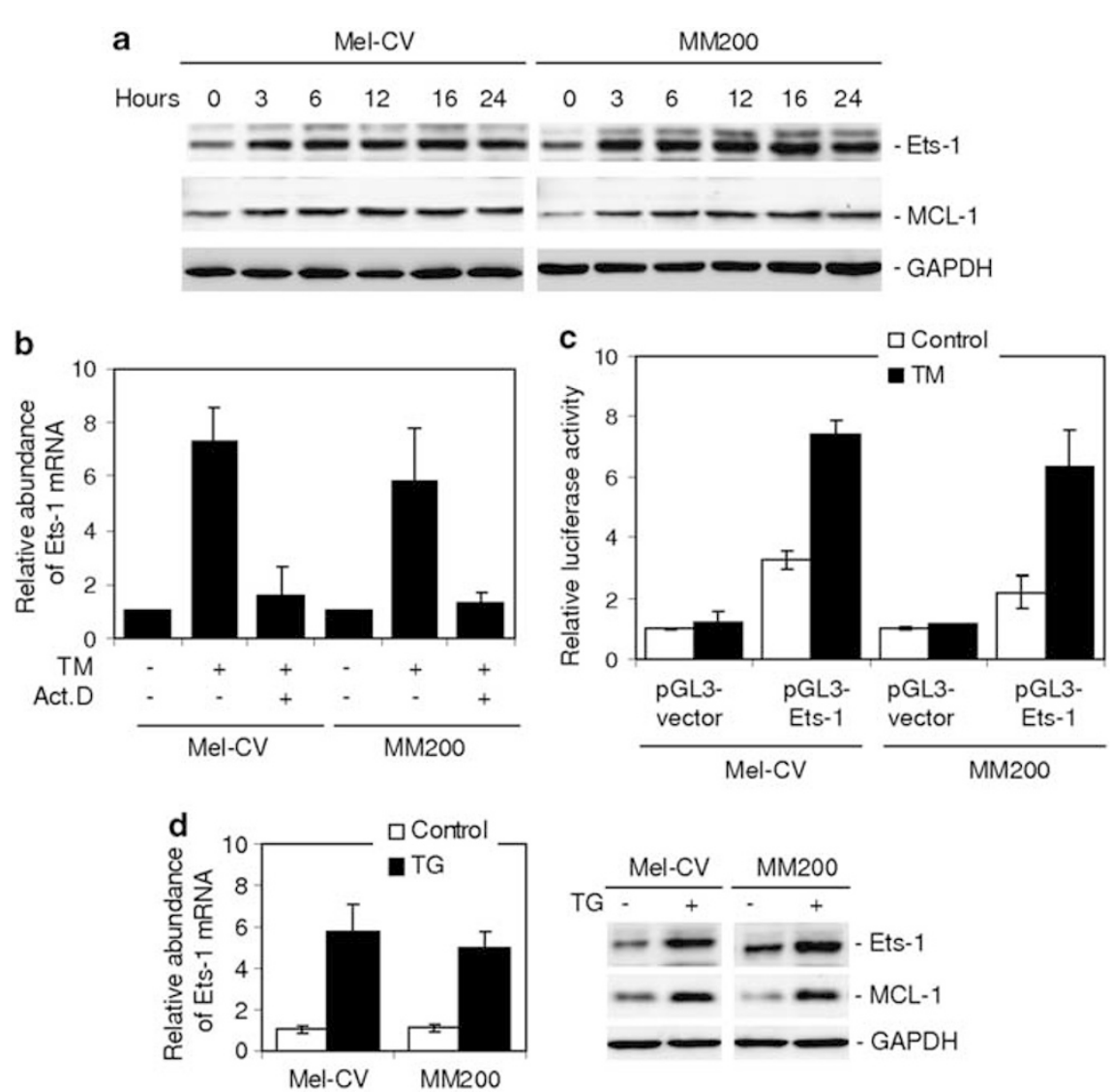

Figure 3 ER stress transcriptionally upregulates Ets-1 in melanoma cells. (a) Whole cell lysates from Mel-CV and MM200 cells with or without treatment with TM $(3 \mu \mathrm{M})$ for indicated periods were subjected to western blot analysis. (b) Mel-CV and MM200 cells with or without pretreatment with actinomycin D $(3 \mu \mathrm{g} / \mathrm{ml})$ for $1 \mathrm{~h}$ were treated with TM $(3 \mu \mathrm{M})$ for $16 \mathrm{~h}$. Total RNA was subjected to qPCR analysis for Ets-1 mRNA expression. The relative abundance of mRNA expression before treatment was arbitrarily designated as 1 . (c) A luciferase reporter containing a $2.5 \mathrm{~kb}$ fragment of the Ets-1 promoter was constructed and transiently transfected into Mel-CV and MM200 cells. After $24 \mathrm{~h}$, cells were treated with TM $(3 \mu \mathrm{M})$ for a further $16 \mathrm{~h}$ followed by measurement of the luciferase activity. (d) Mel-CV and MM200 cells were treated with TG $(1 \mu \mathrm{M}) 16 \mathrm{~h}$. Left panel: total RNA was subjected to qPCR analysis for Ets-1 mRNA expression. The relative abundance of mRNA expression in parental cells was arbitrarily designated as 1. Right: whole cell lysates were subjected to western blot analysis. Bars, s.e. $(n=3)$.

TM upregulated Ets-1 mRNA levels, which could be inhibited by pretreatment with actinomycin D (Figure $3 b$ ), indicating that this was due to a transcriptional increase, rather than a change in the mRNA stability. To confirm transcriptional activation of Ets-1 by TM in melanoma cells, a luciferase reporter containing a $2.5 \mathrm{~kb}$ fragment of the Ets-1 promoter was constructed and transiently transfected into Mel-CV and MM200 cells, as described previously (Jorcyk et al., 1997; Wagner et al., 2008). As shown in Figure 3c, treatment with $\mathrm{TM}$ induced significant increases in transcriptional activity of the reporter in both cell lines $(P<0.05$, two-tailed Student's $t$-test $)$. Upregulation of Ets-1 by ER stress was confirmed in Mel-CV and MM200 cells treated with TG (Figure 3d), and in another four melanoma cell lines treated with TM (Supplementary Figure S4). Notably, treatment with TM did not cause any increase in a melanocyte line (Supplementary Figure S4).

We next tested if inhibition of Ets-1 expression blocks ER stress-induced upregulation of Mcl-1. Knockdown of Ets-1 by Small RNA interference (siRNA) inhibited the transcriptional response of the luciferase reporter containing the Ets-1 binding site (pGL3-Mcl-1-205/ $+10)$ even in the presence of TM $(P<0.05$, two-tailed Student's $t$-test) (Figures $4 \mathrm{a}, \mathrm{b}$ and Supplementary Figure S5). Consistently, Ets-1 siRNA significantly inhibited upregulation of the endogenous Mcl-1 mRNA by TM in both cell lines $(P<0.05$, two-tailed Student's $t$-test) (Figure 4b).

To further confirm the role of Ets-1 in transcriptional upregulation of Mcl-1, we transfected complimentary DNA encoding Ets-1 with or without co-transfection of the luciferase reporters pGL3-Mcl-1-205/+10 into MM200 and Mel-CV cells (Figures 4c and d). Overexpression of Ets-1 markedly enhanced the transcriptional activity of the reporters $(P<0.05$, two-tailed Student's $t$-test), and as anticipated, also increased the endogenous Mcl-1 mRNA expression in both cell lines (Figure 4d).

Upregulation of Ets-1 by ER stress is mediated by XBP-1 To examine the signaling pathways of the UPR responsible for upregulation of Ets-1 in melanoma cells under ER stress, we established melanoma cell lines 

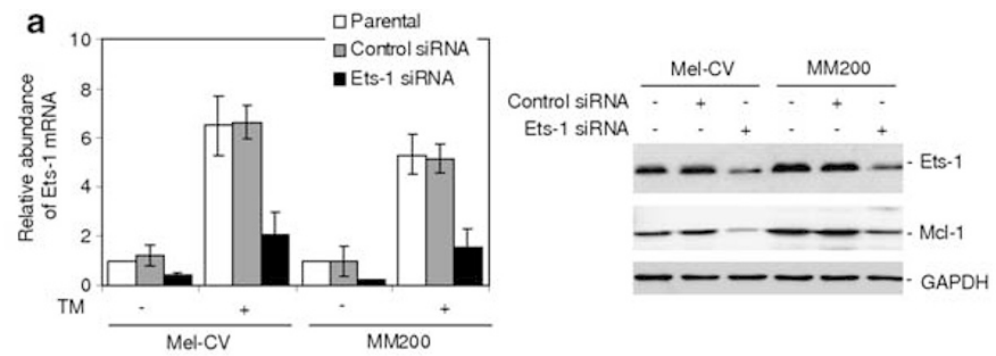

b

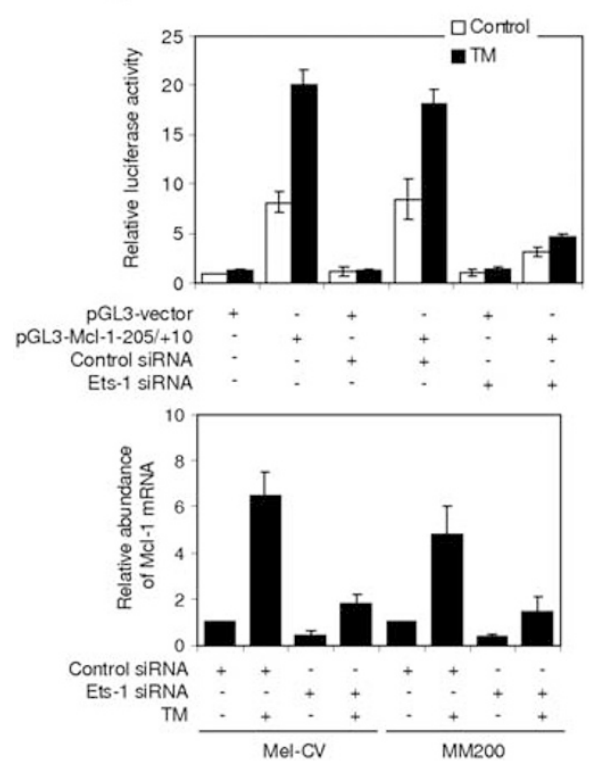

C

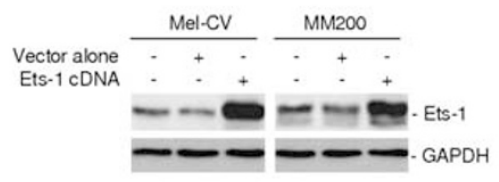

d
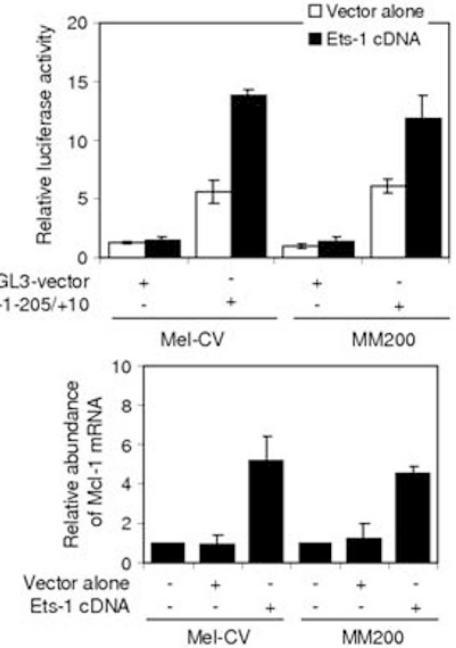

Figure 4 Ets-1 is responsible for transcriptional upregulation of Mcl-1 by ER stress in melanoma cells. (a) Mel-CV and MM200 cells were transfected with the control and Ets-1 small RNA interference (siRNA), respectively. Left panel: After $24 \mathrm{~h}$, cells were treated with TM $(3 \mu \mathrm{M})$ for a further $16 \mathrm{~h}$. Total RNA was subjected to qPCR analysis for Ets-1 mRNA expression. The relative abundance of mRNA expression in parental cells was arbitrarily designated as 1 . Right panel: After $24 \mathrm{~h}$, whole cell lysates were subjected to western blot analysis. Quantitation of the bands showed that the Ets-1 siRNA inhibited Ets-1 expression by 78 and $82 \%$ in Mel-CV and MM200 cells, respectively. (b) Upper panel: Mel-CV cells were co-transfected with the control and Ets-1 siRNA and the pGL3-basicbased luciferase reporter constructs, pGL3-vector and pGL3-Mcl-1-205/ + 10 of the Mcl-1 promoter, respectively. After $24 \mathrm{~h}$, cells were treated with the vehicle control (control) or TM $(3 \mu \mathrm{M})$ for a further $16 \mathrm{~h}$, followed by measurement of the luciferase activity. Lower panel: Mel-CV and MM200 cells were transfected with the control and Ets-1 siRNA. After $24 \mathrm{~h}$, cells were treated with TM $(3 \mu \mathrm{M})$ for a further $16 \mathrm{~h}$. Total RNA was subjected to qPCR analysis for Mcl-1 mRNA expression. The relative abundance of mRNA expression in cells transfected with the control siRNA without treatment with TM was arbitrarily designated as 1 . (c) Mel-CV and MM200 cells were transiently transfected with the vector alone or complimentary DNA encoding Ets-1. After $24 \mathrm{~h}$, whole cell lysates were subjected to western blot analysis. (d) Upper panel: Mel-CV and MM200 cells co-transfected with complimentary DNA encoding Ets-1 and the pGL3-basic-based luciferase reporter constructs, pGL3-vector and pGL3-Mcl-1-205/+10 of the Mcl-1 promoter, respectively. After $24 \mathrm{~h}$, the luciferase activity was measured. Lower panel: Mel-CV and MM200 cells were transiently transfected with the vector alone or complimentary DNA encoding Ets-1. After $24 \mathrm{~h}$, total RNA was subjected to qPCR analysis for Mcl-1 mRNA expression. The relative abundance of mRNA expression in cells with mock transfection was arbitrarily designated as 1 . Bars, s.e. $(n=3)$.

deficient in expression of the three proximal transducers of the UPR, IRE1 $\alpha$, ATF6 and PERK, with short hairpin RNA (shRNA) knockdown. Figure 5a shows that deficiency in IRE1 $\alpha$ or ATF6, but not deficiency in PERK, partially inhibited upregulation of Ets-1 by TM. Consistently, IRE1 $\alpha$ or ATF6 deficiency recapitulated partially the effect of siRNA knockdown of Ets-1 on the expression of Mcl-1 at the mRNA level (Figure 5b).

The IRE1 $\alpha$ and ATF6 pathways of the UPR converge on XBP-1 (Harding et al., 2002; Zhang and Kaufman, 2004; Schroder and Kaufman, 2005). We therefore examined if XBP-1 has a role in ER stress-induced upregulation of Ets-1 in Mel-CV and MM200 cells, with XBP-1 stably knocked down by shRNA. Figure 5c shows that deficiency in XBP-1 inhibited upregulation of Ets-1 by TM, indicative of a critical role of XBP-1 in upregulation of Ets-1 in melanoma cells under ER stress. Similar to IRE1 $\alpha$ or ATF6 deficiency, inhibition of XBP-1 by shRNA recapitulated, in part, the effect of inhibition of Ets-1 by siRNA on the expression of Mcl-1 mRNA (Supplementary Figure S6).

We examined if $\mathrm{XBP}-1$ as a transcription factor is directly responsible for transcriptional upregulation 

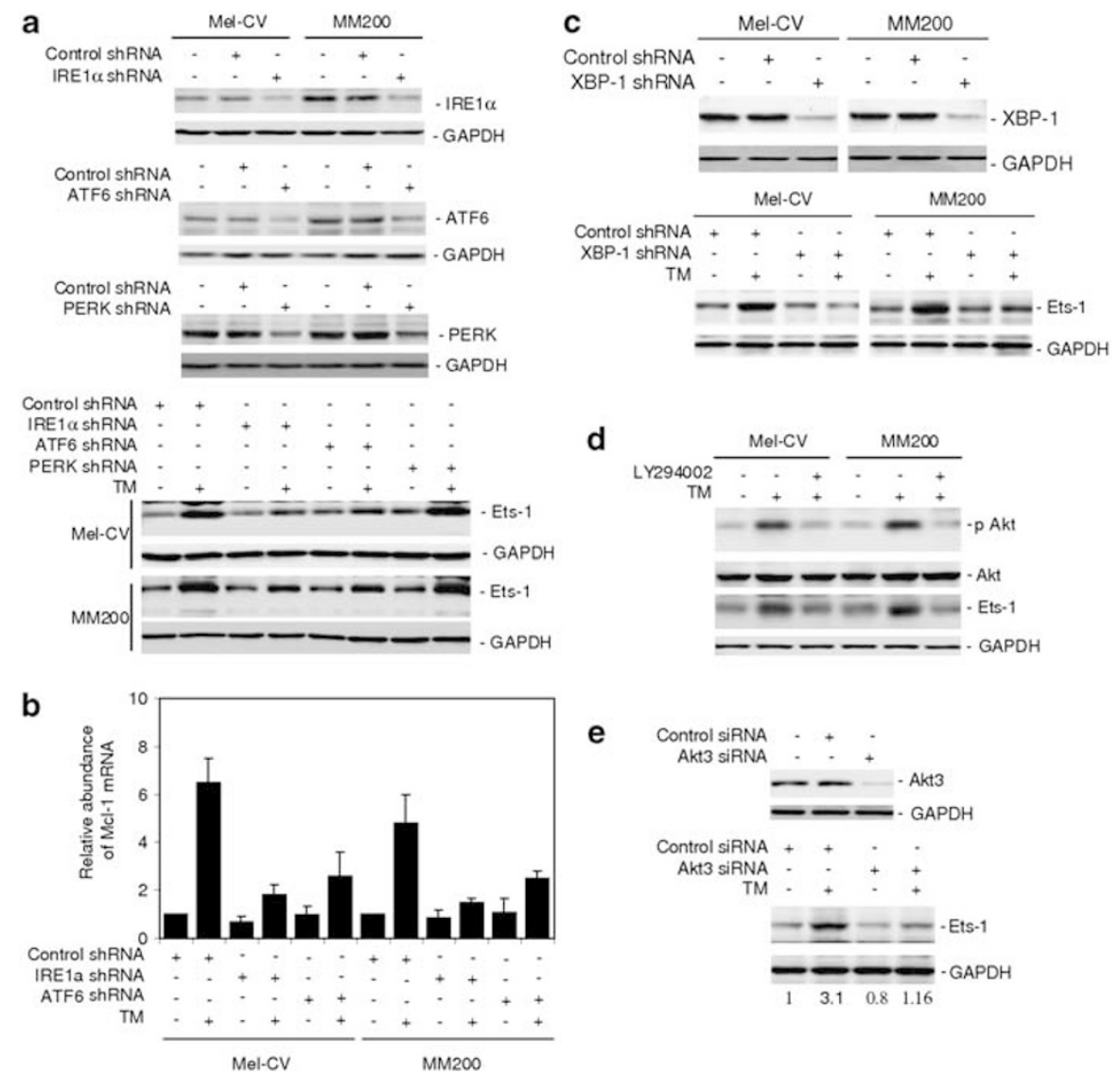

Figure 5 XBP-1 mediates upregulation of Ets-1 in melanoma cells under ER stress. (a) Mel-CV and MM200 cells were transfected with the control, IRE1 $\alpha$, ATF6 and PERK shRNA, respectively. Upper panel: whole cell lysates were subjected to western blot analysis. Quantitation of the bands showed that the IRE1 $\alpha$, ATF6 and PERK shRNA inhibited IRE1 $\alpha$, ATF6 and PERK expression by 71 and $70 \%, 67$ and $75 \%, 76$ and 78\%, in Mel-CV and MM200 cells, respectively. Lower panel: whole cell lysates from cells treated with or without TM $(3 \mu \mathrm{M})$ for $16 \mathrm{~h}$ were subjected to western blot analysis. (b) Mel-CV and MM200 cells with or without IRE1 $\alpha$ and ATF6 knocked down by shRNA, respectively, were treated with TM (3 $\mu \mathrm{M})$ for $16 \mathrm{~h}$. Total RNA was subjected to qPCR analysis for Mcl-1 mRNA expression. The relative abundance of mRNA expression in cells carrying the control shRNA without treatment with TM was arbitrarily designated as 1. (c) Mel-CV and MM200 cells were transfected with the control and XBP-1 shRNA, respectively. Upper panel: whole cell lysates were subjected to western blot analysis. Quantitation of the bands showed that the XBP-1 shRNA inhibited XBP-1 expression by 82 and $85 \%$ in Mel-CV and MM200 cells, respectively. Lower panel: whole cell lysates from cells treated with or without TM $(3 \mu \mathrm{M})$ for $16 \mathrm{~h}$ were subjected to western blot analysis. (d) Mel-CV and MM200 cells with or without pretreatment with the PI3k inhibitor LY294002 $(20 \mu \mathrm{M})$ for $1 \mathrm{~h}$ were treated with TM $(3 \mu \mathrm{M})$ for a further $16 \mathrm{~h}$. Whole cell lysates were subjected to western blot analysis. (e) MM200 cells were transfected with the control or Akt3 small RNA interference (siRNA). Upper panel: After $24 \mathrm{~h}$, whole cell lysates were subjected to western blot analysis. Quantitation of the bands showed that the Akt3 siRNA inhibited Akt3 expression by $90 \%$. Lower panel: After $24 \mathrm{~h}$, cells were treated with TM $(3 \mu \mathrm{M})$ for a further $16 \mathrm{~h}$. Whole cell lysates were subjected to western blot analysis. The intensity of the Ets-1 bands was quantitated and the value in cells transfected with the control siRNA without treatment with TM was arbitrarily designated as 1 . The relative expression level of Ets-1 in each sample was depicted below the corresponding blot.

of Ets-1 in melanoma cells induced by ER stress (Harding et al., 2002; Zhang and Kaufman, 2004; Schroder and Kaufman, 2005). Although examination of the flanking genomic DNA region identified a potential XBP-1 binding site located 1775-1793 bp upstream of the Ets-1 transcription start site (data not shown), there was no binding of XBP-1 to the Ets-1 promoter as shown in both EMSA and ChIP assays (data not shown).

We have previously found that XBP-1 mediates ER stress-induced activation of Akt in melanoma cells, which is known to have a role in regulation of Mcl-1 transcription (Jiang et al., 2009b). We therefore tested the relationship between the activation of Akt and upregulation of Ets-1. As shown in Figure 5d, treatment with the PI3k inhibitor LY294002 blocked activation of Akt and inhibited upregulation of Ets-1 induced by TM. Notably, knockdown of Akt3, the major isoform of Akt in melanoma (Jiang et al., 2009b), reduced the basal levels of Ets-1, suggesting that the PI3k/Akt pathway may have a role in regulating constitutive expression of Ets-1 in melanoma cells (Figure 5e). Although the addition of TM caused a threefold increase in Ets-1 in cells transfected with the control siRNA, there was only a 1.4-fold increase in cells with Akt3 knocked down (Figure 5e), indicating that activation of Akt downstream of XBP-1 is involved in ER stress-induced upregulation of Ets-1. 
Ets-1 protects melanoma cells from ER stress-induced apoptosis

Because Mcl-1 has a critical role in protection of melanoma cells from ER stress-induced apoptosis (Hersey and Zhang, 2008; Jiang et al., 2008), we examined whether Ets-1 has a similar role. As expected, inhibition of Ets-1 by siRNA rendered Mel-CV and MM200 cells sensitive to apoptosis induced by TM (Figure 6a), which was confirmed by induction of caspase-3 activation (Figure 6b). This sensitization could be blocked by overexpression of Mcl-1, and was not further enhanced in melanoma cells deficient in Mcl-1 (Figures 6c, d and Supplementary Figure S7), suggesting that it was mediated by downregulation of Mcl-1. Similarly, inhibition of Ets-1 also sensitized Mel-CV and MM200 cells to apoptosis induced by TG (Supplementary Figure S8).

We next examined the effect of inhibition of Ets-1 on responses to ER stress in fresh melanoma isolates, which may more closely reflect the in vivo situation where the cells are resistant to ER-stress-induced apoptosis (Nguyen et al., 2001; Jiang et al., 2007). Freshly isolated melanoma cells expressed in general relatively high levels of Ets-1 in comparison with cultured melanocytes (Supplementary Figure S9A). As reported before (Liu et al., 2009; Jiang et al., 2009b), neither TM nor TG induced significant apoptosis in fresh melanoma isolates $(<20 \%$ apoptotic cells) (Figure $6 \mathrm{e}$ ). However, when Ets-1 was knocked down, there were significant increases in induction of apoptosis by the compounds $(P<0.05$, respectively, two-tailed Student's $t$-test $)$ (Figure 6e and Supplementary Figure S9B).

\section{Discussion}

The above results extend our previous finding that Mcl-1 is upregulated in melanoma cells under ER stress, by showing that Ets-1 is responsible for the transcriptional increase in Mcl-1 (Jiang et al., 2008). They show that Ets- 1 is also upregulated downstream of the IRE1 $\alpha /$ XBP-1 branch of the UPR, and that this is associated with activation of the PI3k/Akt pathway. Moreover, the results demonstrate a role of Ets-1 in protection of melanoma cells against ER stress-induced apoptosis, which recapitulates, at least in part, the effect of inhibition of Mcl-1 on sensitivity of melanoma cells to apoptosis induced by ER stress.

Mcl-1 is of particular importance in melanoma, as its expression increases with melanoma progression, is associated with poorer prognosis, and is a key adaptive mechanism of melanoma cells to cellular stress conditions including ER stress (Wang et al., 2007; Zhuang et al., 2007, 2009, 2010). Although Mcl-1 is regulated by a number of signaling pathways (Epling-Burnette et al., 2001; Harding et al., 2002; Iglesias-Serret et al., 2003; Wang et al., 2003; Isomoto et al., 2005; Piret et al., 2005; Warr and Shore, 2008), upregulation of Mcl-1 by the UPR appears to be characteristic of melanoma cells, in that Mcl-1 is commonly downregulated by ER stress in other cell types (Fritsch et al., 2007). Our previous results have shown that upregulation of $\mathrm{Mcl}-1$ in melanoma cells upon ER stress involves a transcriptional increase (Jiang et al., 2008). In the present study, we identified Ets-1 as a key transcription factor that activates Mcl-1 transcription during ER stress in melanoma cells. This was demonstrated by (1) the shortest fragment of the $\mathrm{Mcl}-1$ promoter responsive to ER stress contained a consensus binding site of Ets; (2) Ets-1 bound directly to this site, which was enhanced by induction of ER stress; (3) mutations in this site diminished ER stress-induced activation of the Mcl-1 promoter; and (4), knockdown of Ets-1 inhibited activation of Mcl-1 transcription induced by ER stress, whereas overexpression of Ets-1 enhanced Mcl-1 promoter activity leading to increased Mcl-1 expression. Even though we cannot exclude involvement of changes in the $\mathrm{Mcl}-1$ protein turnover rate, these results clearly demonstrate an important role of Ets-1-mediated increases in Mcl-1 at the transcriptional level in its upregulation induced by ER stress in melanoma cells.

Ets-1 is expressed at high levels in many types of cancers, and is involved in many biological processes in cancer cells such as cell growth and survival (Davidson et al., 2001; Span et al., 2002; Dittmer, 2003; Hahne et al., 2008). In melanoma, the levels Ets-1 were similarly found to be higher than those in benign melanocytic lesions and melanocytes, and to increase with progression of the disease (Poser and Bosserhoff, 2004; Rothhammer et al., 2004; Torlakovic et al., 2004). Consistently, the results in this study also showed that Ets-1 was commonly expressed at high levels in fresh melanoma isolates. Although the role of Ets-1 in regulation of apoptosis may vary between different cell types (Bories et al., 1995; Teruyama et al., 2001), our finding that Ets-1 was responsible for transcriptional upregulation of Mcl-1 in melanoma cells subjected to ER stress indicates that it is critical in protection of melanoma cells against ER stress-induced apoptosis. It is conceivable that, like Mcl-1, Ets-1 may also contribute to resistance of melanoma cells to apoptosis induced by agents other than those that induce ER stress (Liu et al., 2009; Jiang et al., 2009c).

An important finding of this study is upregulation of Ets-1 by ER stress in melanoma cells. This appeared to be due to a transcriptional mechanism. Upregulation of Ets-1 by ER stress was partially inhibited in melanoma cells deficient in IRE1 $\alpha$ or ATF6, indicating that these branches of the UPR are involved in the ER stress-triggered increase in Ets-1 in melanoma cells. On activation, the RNase activity of IRE $1 \alpha$ cleaves XBP1 mRNA, generating a splicing variant that encodes a potent transcription factor (Harding et al., 2002; Zhang and Kaufman, 2004; Schroder and Kaufman, 2005). As a transcription factor, activated ATF6 activates transcription of UPR target genes including $X B P-1$ (Harding et al., 2002; Zhang and Kaufman, 2004; Schroder and Kaufman, 2005). The convergence of the IRE1 $\alpha$ and ATF6 pathways on XBP-1 suggests that XBP-1 may be involved in upregulation of Ets- 1 by the UPR. This was validated in melanoma cells with XBP-1 

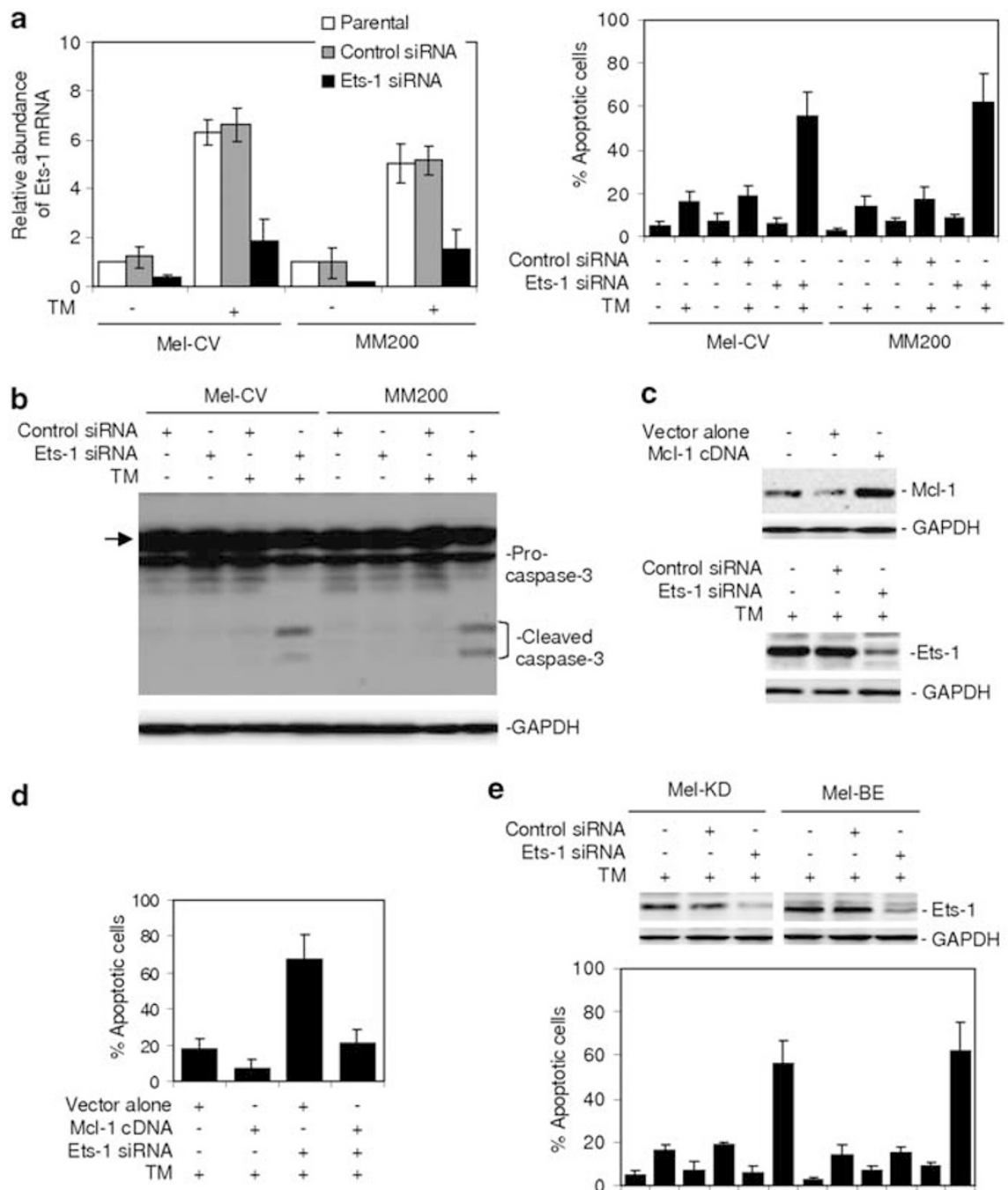

C
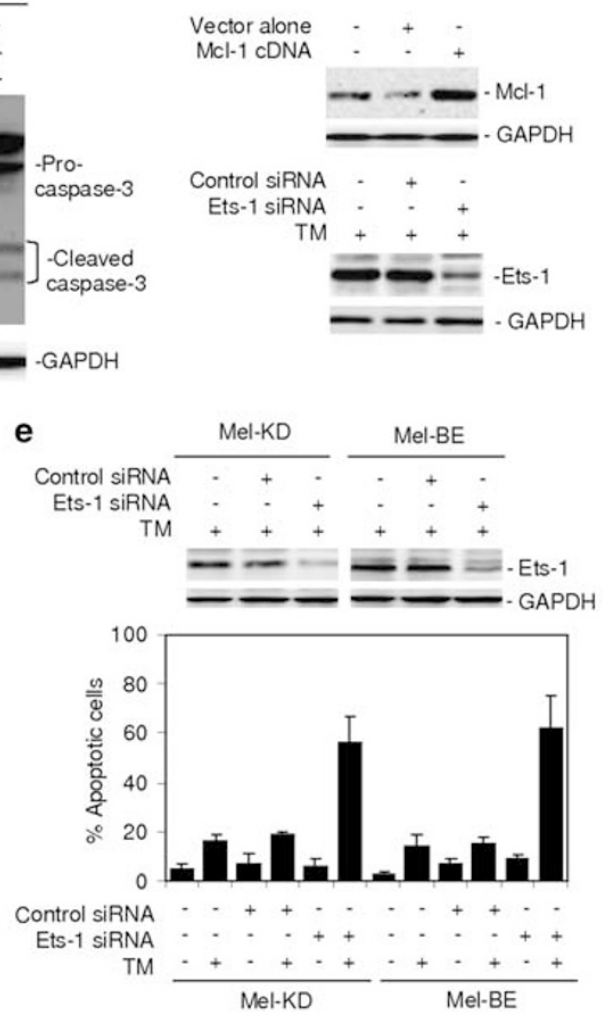

Figure 6 Inhibition of Ets-1 renders cultured melanoma cells and fresh melanoma isolates sensitive to ER stress-induced apoptosis. (a) Mel-CV and MM200 cells were transfected with the control and Ets-1 small RNA interference (siRNA), respectively. Left panel: After $24 \mathrm{~h}$, cells were treated with TM $(3 \mu \mathrm{M})$ for a further $16 \mathrm{~h}$. Total RNA was subjected to qPCR analysis for Ets-1 mRNA expression. The relative abundance of mRNA expression in parental cells was arbitrarily designated as 1. Right panel: After $24 \mathrm{~h}$, cells were treated with TM $(3 \mu \mathrm{M})$ for a further $48 \mathrm{~h}$. Apoptotic cells were quantitated by the propidium iodide method. (b) Mel-CV and MM200 cells were transfected with the control and Ets-1 siRNA, respectively. After $24 \mathrm{~h}$, cells were treated with TM (3 $\mu \mathrm{M})$ for a further $36 \mathrm{~h}$. Whole cell lysates were then subjected to western blot analysis. The arrow heads point to a non-specific band generated by the antibody against caspase-3. (c) Upper panel: whole cell lysates from MM200 cells stably transfected with the vector alone or complimentary DNA encoding Mcl-1 were subjected to western blot analysis. Lower panel: MM200 cells stably transfected with complimentary DNA encoding Mcl-1 were transfected with the control or Ets-1 siRNA. After $24 \mathrm{~h}$, whole cell lysates were subjected to western blot analysis. Quantitation of the bands showed that the Ets-1 siRNA inhibited Ets-1 expression by $80 \%$. (d) MM200 cells stably transfected with complimentary DNA encoding Mcl-1 were transfected with the control or Ets-1 siRNA. After $24 \mathrm{~h}$, cells were treated with TM $(3 \mu \mathrm{M})$ for a further $48 \mathrm{~h}$. Apoptotic cells were quantitated by the propidium iodide method. (e) Upper panel: Mel-KD and Mel-BE fresh melanoma isolates were transfected with the control or Ets-1 siRNA. After $24 \mathrm{~h}$, whole cell lysates were subjected to western blot analysis. Quantitation of the bands showed that the Ets-1 siRNA inhibited Ets-1 expression by 82 and $78 \%$ in Mel-KD and Mel-BE cells, respectively. Lower panel: Mel-KD and Mel-BE cells were transfected with the control or Ets-1 siRNA. After $24 \mathrm{~h}$, cells were treated with TM $(3 \mu \mathrm{M})$ for a further $48 \mathrm{~h}$. Apoptotic cells were quantitated by the propidium iodide method. Bars, s.e. $(n=3)$.

knocked down by shRNA. These results were in line with the essential role of XBP-1 in upregulation of Mcl-1, as reported previously (Jiang et al., 2008).

Consistent with our previous finding that activation of the PI3k/Akt pathway by XBP-1 is involved in upregulation of Mcl-1 by the UPR in melanoma cells
(Jiang et al., 2009b), we found in this study that activation of PI3k/Akt downstream of XBP-1 had a role in ER stress-induced upregulation of Ets-1. The mechanism(s) by which the PI3K/Akt pathway mediates regulation of Ets-1 remains to be determined, but it is known that activation of Akt increases the expression of 

2003). Some other transcription factors that have been shown to transcriptionally upregulate Ets- 1 include Sp1, HIF-1 and Ets-1 itself (Majeacuterus et al., 1992; Dittmer, 2003; Hahne et al., 2008).

Our finding that Ets-1 has a role in protection of freshly isolated melanoma cells against ER stressinduced apoptosis is significant, as they may reflect more closely responses of melanoma cells to ER stress in vivo (Nguyen et al., 2001; Jiang et al., 2007). The relatively high levels of Ets-1 expression is conceivably a consequence of activation of the UPR (Zhuang et al., 2009; Jiang et al., 2009c), and a means of adaptation to chronic ER stress conditions encountered by melanoma cells in vivo. Collectively, results in this study reveal a key mechanism responsible for transcriptional upregulation of Mcl-1 by ER stress in melanoma cells, and identify upregulation of Ets- 1 as part of the adaptive mechanism of the cells to ER stress. Ets-1 may therefore be a potential target for the treatment of melanoma in combination with therapeutics that induce ER stress.

\section{Materials and methods}

Cell culture and reagents

Human melanoma cell lines Me4405, ME1007, Mel-CV, Sk-Mel-28, Sk-Mel-110 and MM200 have been described previously and were cultured in Dulbecco's modified Eagle's medium containing $5 \%$ fetal calf serum (Commonwealth Serum Laboratories, Melbourne, VIC, Australia) (Gillespie et al., 2005). DNA for cell line authentication was extracted from all the cell lines while cultured for this study. Individual cell line authentication was confirmed using the AmpFISTR Identifiler PCR Amplification Kit from Applied Biosystems (Mulgrave, VIC, Australia) and GeneMarker V1.91 software (SoftGenetics LLC, State College, PA, USA). A panel of 16 markers was tested, and each cell line had a distinct individual set of markers present. TM and TG were purchased from Sigma-Aldrich (Castle Hill, NSW, Australia). The PI3 K inhibitor, 2-(4-morpholinyl)-8-phenyl-4H-1-benzopyran-4-one (LY294002), was purchased from Calbiochem (Kilsyth, VIC, Australia). The mouse monoclonal antibodies against Mcl-1 and the rabbit polyclonal antibodies (Abs) against Ets-1, Ets-2, c-Rel, XBP-1, GRP78, IRE1a, ATF6 and PERK were purchased from Santa Cruz Biotechnology (Santa Cruz, CA, USA). The rabbit antibodies against Akt, phospho-Akt (Ser473) were from Cell Signaling Technology (Danvers, MA, USA). The rabbit polyclonal antibodies against caspase-3 were from Stressgen Biotechnologies (Victoria, BC, Canada).

\section{Fresh melanoma isolates}

Isolation of melanoma cells from fresh surgical specimens was carried out as described previously (Jiang et al., 2010).

\section{Apoptosis}

Quantitation of apoptotic cells was carried out using propidium iodide as described elsewhere (Jiang et al., 2010; Yang et al., 2010).

\section{Western blot analysis}

Western blot analysis was carried out as described previously (Jiang et al., 2010; Yang et al., 2010). The intensity of bands

was quantitated relative to corresponding GAPDH bands with the Bio-Rad VersaDoc image system (Bio-Rad, Regents Park, NSW, Australia).

Quantitative reverse transcription and real-time-PCR Quantitative reverse transcription and real-time PCR was performed as described previously (Jiang et al., 2010; Yang et al., 2010). The primers used for PCR are as follows: ETS1: sense, 5'-GTCGTGGTAAACTCGG-3', anti-sense, 5'-CAGC AGGAATGACAGG-3'; Mcl-1: sense, 5'-CTTACGACGGG TTGGG-3', anti-sense, 5'-GGTTCGATGCAGCTTTCTTG G-3'; c-Rel: sense, 5' -TTGGACAAGAACGCAGAC-3', antisense, 5'-CAGGAGGAAGAGCAGTCGT-3'.

\section{SiRNA}

The siRNA constructs used were obtained as the siGENOME SMARTpool reagents (Dharmacon, Lafayette, CO, USA), c-Rel siGENOME SMARTpool (M-004768-01-0010), Ets-1 siGENOME SMARTpool (M-003887-00-0010), AKT3 siGENOME SMARTpool (M-003002-02-0010), and siGENO ME Non-targeting SiRNA pool (D-001206-13-20). Transfection of siRNA pools was carried out as described previously (Jiang et al., 2010; Yang et al., 2010).

$\operatorname{sh} R N A$

Sigma MISSION Lentiviral Transduction Particles for shRNA-mediated knockdown of Mcl-1 were purchased from Sigma-Aldrich and used as described previously (Castle Hill, NSW, Australia) (Jiang et al., 2008).

\section{Luciferase-reporter constructs}

The Mcl-1 promoter sequence from $1300 \mathrm{bp}$ upstream to $10 \mathrm{bp}$ downstream of the human Mcl-1 gene transcription start site was cloned by genomic PCR using human genomic DNA as a template. Deletions of the promoter were generated by PCR with $5^{\prime}$ primers and a fixed $3^{\prime}$ primer. The sequences of these forward primers were: 5'-GCTAGCAACTGATCAATGTAC TTTGTAATCT-3' $(-1300 / 10), \quad 5^{\prime}$-GCTAGCATTTGGTAAA AAACCTCTGGCG-3'(-300/10), 5'-GCTAGCTCGGAGCC GCCGTTAC-3'(-224/10), 5'-GCTAGCCAGAGCCTCCGA AGACCGG-3'(-205/10), 5'-GCTAGCTCAGGCCCCGGCT CAGG -3'(-175/10), 5' - GCTAGCCTGCCGCCCCTTTCCC CTTTT-3'(-65/10). The reverse primer was: $5^{\prime}$-CCCCAAGCT TGCCTACGGGGTGGCGCCAGCGAAC- $3^{\prime}$. Mutagenesis of the Ets1 binding site was performed by PCR using oligonucleotides carrying mutations at the presumed Ets 1 core recognition sites, in combination with the anti-sense primer $(+10)$. These Mcl-1 promoter fragments were cloned into promoter-less luciferase reporter plasmid pGL3-Basic Luciferase Vector (Promega, Madison, WI, USA). A $2.5 \mathrm{~kb}$ fragment of the Ets-1 promoter was subcloned into the pGL3-Basic Luciferase Vector as described (Jorcyk et al., 1997; Wagner et al., 2008). Cells were transiently transfected with luciferase constructs together with the pRL-TK vector as a control for transfection efficiency. The luciferase activity was measured using the Dual Luciferase Reporter Assay System (Promega) by Synergy 2 multi-detection microplate reader (BioTek, Vinooski, VT, USA).

\section{EMSA}

Nuclear extracts were prepared using NE-PER Nuclear Extraction Reagents Kit (Pierce, Rockford, IL, USA). The DNA-protein binding was detected using the Light-Shift chemiluminescent EMSA kit (Pierce). Binding reactions were performed by adding $2 \mu \mathrm{g}$ of the nuclear extracts to a mixture 
containing $40 \mathrm{fmol}$ of biotin-labeled, double-stranded probes (5'-CAGAGCCTCCGAAGACCGGAAGGCCCCGCT- $3^{\prime}$ ) in $20 \mathrm{il}$ of binding buffer. Antibodies were added to aliquots of extracts for $30 \mathrm{~min}$ before the addition of the reaction mixture. Competition reaction mixtures contained a 100-fold molar excess of non-labeled double-stranded oligo DNAs. The mixtures were then resolved by polyacrylamide gel electrophoresis and probe binding visualized by streptavidin conjugated with horseradish peroxidase.

\section{ChIP analysis}

Analyses were performed using the ChIP Assay Kit (Upstate, Lake Placid, NY, USA). Briefly, cells were cross-linked using $1 \%$ formaldehyde at $37^{\circ} \mathrm{C}$ for $10 \mathrm{~min}$. After washing, cells were resuspended in $200 \mu \mathrm{l}$ SDS lysis buffer. DNA was sheared to small fragments of 200 to $1000 \mathrm{bp}$ by sonication. The recovered supernatant was incubated with antibodies overnight at $4{ }^{\circ} \mathrm{C}$ with rotation. After washing, the precipitated protein-DNA complexes were dissolved in $1 \times \mathrm{TE}$ buffer and incubated at $65^{\circ} \mathrm{C}$ for $4 \mathrm{~h}$. DNA was then purified with phenol/chloroform, and a fraction was used as PCR template to detect the presence of the promoter sequences between -205 and +10 of Mcl-1 using Mcl-1-specific primers: 5'-CAGAGC CTCCGAAGACCGG-3' (forward); 5'-CCTACGGGGTGG CGCCAGCGAAC-3'(reverse).

\section{Conflict of interest}

The authors declare no conflict of interest.

\section{Acknowledgements}

This work was supported by the NSW State Cancer Council, Cancer Institute NSW, and National Health and Medical Research Council (NHMRC), Australia. L Dong is a recipient of Australia-China Exchange Fellowship of NHMRC. XD Zhang is supported by a senior research fellowship of NHMRC.

\section{References}

Akgul C, Turner PC, White MR, Edwards SW. (2000). Functional analysis of the human MCL-1 gene. Cell Mol Life Sci 57: 684-691.

Bories JC, Willerford DM, Grévin D, Davidson L, Camus A, Martin P et al. (1995). Increased T-cell apoptosis and terminal B-cell differentiation induced by inactivation of the Ets-1 proto-oncogene. Nature 377: 635-638.

Chen C, Edelstein LC, Gélinas C.. (2000). The Rel/NF-kappaB family directly activates expression of the apoptosis inhibitor Bcl-x(L). Mol Cell Biol 20: 2687-2695.

Cory S, Adams JM.. (2002). The Bcl2 family: regulators of the cellular life-or-death switch. Nat Rev Cancer 2: 647-656.

Davidson B, Risberg B, Goldberg I, Nesland JM, Berner A, Tropé CG et al. (2001). Ets-1 mRNA expression in effusions of serous ovarian carcinoma patients is a marker of poor outcome. Am J Surg Pathol 25: $1493-1500$.

Dittmer J. (2003). The biology of the Ets 1 proto-oncogene. Mol Cancer 2: 29.

Elbein AD. (1987). Inhibitors of the biosynthesis and processing of N-linked oligosaccharide chains. Annu Rev Biochem 56: 497-534.

Epling-Burnette PK, Liu JH, Catlett-Falcone R, Turkson J, Oshiro M, Kothapalli R et al. (2001). Inhibition of STAT3 signaling leads to apoptosis of leukemic large granular lymphocytes and decreased Mcl-1 expression. $J$ Clin Invest 107: 351-362.

Fritsch RM, Schneider G, Saur D, Scheibel M, Schmid RM.. (2007). Translational repression of MCL-1 couples stress-induced eIF2 alpha phosphorylation to mitochondrial apoptosis initiation. $J$ Biol Chem 282: 22551-22562.

Gillespie S, Zhang XD, Hersey P. (2005). Variable expression of protein kinase $\mathrm{C}$ epsilon in human melanoma cells regulates sensitivity to TRAIL-induced apoptosis. Mol Cancer Ther 4: 668-676.

Hahne JC, Okuducu AF, Sahin A, Fafeur V, Kiriakidis S, Wernert N. (2008). The transcription factor ETS-1: its role in tumour development and strategies for its inhibition. Mini Rev Med Chem 8: 1095-1105.

Harding HP, Calfon M, Urano F, Novoa I, Ron D. (2002). Transcriptional and translational control in the mammalian unfolded protein response. Annu Rev Cell Dev Biol 18: 575-599.

Hersey P, Zhuang L, Zhang XD. (2006). Current strategies in overcoming resistance of cancer cells to apoptosis melanoma as a model. Int Rev Cytol 251: 131-158.

Hersey P, Zhang XD. (2008). Adaptation to ER stress as a driver of malignancy and resistance to therapy in human melanoma. Pigment Cell Melanoma Res 21: 358-367.
Iglesias-Serret D, Piqué M, Gil J, Pons G, López JM. (2003). Transcriptional and translational control of Mcl-1 during apoptosis. Arch Biochem Biophys 417: 141-152.

Isomoto H, Kobayashi S, Werneburg NW, Bronk SF, Guicciardi ME, Frank DA et al. (2005). Interleukin 6 upregulates myeloid cell leukemia-1 expression through a STAT3 pathway in cholangiocarcinoma cells. Hepatology 42: 1329-1338.

Jiang CC, Wroblewski D, Yang F, Hersey P, Zhang XD. (2009a). Human melanoma cells under endoplasmic reticulum stress are more susceptible to apoptosis induced by the $\mathrm{BH} 3$ mimetic obatoclax. Neoplasia 11: 945-955.

Jiang CC, Lucas K, Avery-Kiejda KA, Wade M, deBock CE, Thorne $\mathrm{RF}$ et al. (2008). Up-regulation of Mcl-1 is critical for survival of human melanoma cells upon endoplasmic reticulum stress. Cancer Res 68: 6708-6717.

Jiang CC, Yang F, Thorne RF, Zhu BK, Hersey P, Zhang XD. (2009b). Human melanoma cells under endoplasmic reticulum stress acquire resistance to microtubule-targeting drugs through XBP-1-mediated activation of Akt. Neoplasia 11: 436- 447.

Jiang CC, Chen LH, Gillespie S, Wang YF, Kiejda KA, Zhang XD et al. (2007). Inhibition of MEK sensitizes human melanoma cells to endoplasmic reticulum stress-induced apoptosis. Cancer Res 67: 9750-9761.

Jiang CC, Mao ZG, Avery-Kiejda KA, Wade M, Hersey P, Zhang XD. (2009c). Glucose-regulated protein 78 antagonizes cisplatin and adriamycin in human melanoma cells. Carcinogenesis 30: $197-204$

Jiang CC, Lai F, Tay KH, Croft A, Rizos H, Becker TM et al. (2010). Apoptosis of human melanoma cells induced by inhibition of BRAFV600E involves preferential splicing of BimS. Cell Death Dis 1: e69; doi:10.1038/cddis.2010.48.

Jorcyk CL, Garrett LJ, Maroulakou IG, Watson DK, Green JE. (1997). Multiple regulatory regions control the expression of Ets-1 in the developing mouse: vascular expression conferred by intron I. Cell Mol Biol (Noisy-le-grand) 43: 211-225.

Li R, Pei H, Watson DK, Papas TS. (2000). EAP1/Daxx interacts with ETS1 and represses transcriptional activation of ETS1 target genes. Oncogene 19: 745-753.

Liu H, Jiang CC, Lavis CJ, Croft A, Dong L, Tseng HY et al. (2009). 2-Deoxy-D-glucose enhances TRAIL-induced apoptosis in human melanoma cells through XBP-1-mediated up-regulation of TRAIL-R2. Mol Cancer 8: 122 . 
Majeacuterus MA, Bibollet-Ruche F, Telliez JB, Wasylyk B, Bailleul B.. (1992). Serum, AP-1 and Ets-1 stimulate the human ets-1 promoter. Nucleic Acids Res 20: 2699-2703.

Mhaidat NM, Zhang XD, Jiang CC, Hersey P.. (2007). Docetaxelinduced apoptosis of human melanoma is mediated by activation of c-Jun NH2-terminal kinase and inhibited by the mitogen-activated protein kinase extracellular signal-regulated kinase 1/2 pathway. Clin Cancer Res 13: 1308-1314.

Myers E, Hill AD, Kelly G, McDermott EW, O'Higgins NJ, Buggy Y et al. (2005). Associations and interactions between Ets-1 and Ets-2 and coregulatory proteins, SRC-1, AIB1, and NCoR in breast cancer. Clin Cancer Res 11: 2111-2122.

Nguyen T, Zhang XD, Hersey P. (2001). Relative resistance of fresh isolates of melanoma to tumor necrosis factor-related apoptosisinducing ligand (TRAIL)-induced apoptosis. Clin Cancer Res 7(3 Suppl): 966s-973s.

Piret JP, Minet E, Cosse JP, Ninane N, Debacq C, Raes M et al. (2005). Hypoxia-inducible factor-1-dependent overexpression of myeloid cell factor-1 protects hypoxic cells against tert-butyl hydroperoxide-induced apoptosis. J Biol Chem 280: 9336-9344.

Poser I, Bosserhoff AK.. (2004). Transcription factors involved in development and progression of malignant melanoma. Histol Histopathol 19: 173-188.

Rothhammer T, Hahne JC, Florin A, Poser I, Soncin F, Wernert N et al. (2004). The Ets-1 transcription factor is involved in the development and invasion of malignant melanoma. Cell $\mathrm{Mol}$ Life Sci 61: 118-128.

Sagara Y, Inesi G.. (1991). Inhibition of the sarcoplasmic reticulum $\mathrm{Ca} 2+$ transport ATPase by thapsigargin at subnanomolar concentrations. J Biol Chem 266: 13503-13506.

Sato Y, Teruyama K, Nakano T, Oda N, Abe M, Tanaka K et al. (2001). Role of transcription factors in angiogenesis: Ets-1 promotes angiogenesis as well as endothelial apoptosis. Ann NY Acad Sci 947: $117-123$.

Schwickart M, Huang X, Lill JR, Liu J, Ferrando R, French DM et al. (2010). Deubiquitinase USP9X stabilizes MCL1 and promotes tumour cell survival. Nature 463: 103-107.

Schroder M, Kaufman RJ. (2005). The mammalian unfolded protein response. Annu Rev Biochem 74: 739-789.

Soengas MS, Lowe SW. (2003). Apoptosis and melanoma chemoresistance. Oncogene 22: 3138-3151.

Span PN, Manders P, Heuvel JJ, Thomas CM, Bosch RR, Beex LV et al. (2002). Expression of the transcription factor Ets-1 is an independent prognostic marker for relapse-free survival in breast cancer. Oncogene 21: 8506-8509.

Teruyama K, Abe M, Nakano T, Iwasaka-Yagi C, Takahashi S, Yamada S et al. (2001). Role of transcription factor Ets-1 in the apoptosis of human vascular endothelial cells. J Cell Physiol 188: 243-252.

Torlakovic EE, Bilalovic N, Nesland JM, Torlakovic G, Flørenes VA. (2004). Ets-1 transcription factor is widely expressed in benign and malignant melanocytes and its expression has no significant association with prognosis. Mod Pathol 17: 1400-1406.

Wagner N, Michiels JF, Schedl A, Wagner KD. (2008). The Wilms' tumour suppressor WT1 is involved in endothelial cell proliferation and migration: expression in tumour vessels in vivo. Oncogene 27: 3662-3672.

Wang YF, Jiang CC, Kiejda KA, Gillespie S, Zhang XD, Hersey P. (2007). Apoptosis induction in human melanoma cells by inhibition of MEK is caspase-independent and mediated by the Bcl-2 family members PUMA, Bim, and Mcl-1. Clin Cancer Res 13: 4934-4942.

Wang JM, Lai MZ, Yang-Yen HF. (2003). Interleukin-3 stimulation of mcl-1 gene transcription involves activation of the PU.1 transcription factor through a p38 mitogen-activated protein kinase-dependent pathway. Mol Cell Biol 23: 1896-1909.

Warr MR, Shore GC. (2008). Unique biology of Mcl-1: therapeutic opportunities in cancer. Curr Mol Med 8: 138-147.

Yang F, Tay KH, Dong L, Thorne RF, Jiang CC, Yang E et al. (2010). Cystatin B inhibition of TRAIL-induced apoptosis is associated with the protection of FLIP(L) from degradation by the E3 ligase itch in human melanoma cells. Cell Death Differ 17: 1354-1367.

Zhang K, Kaufman RJ. (2004). Signaling the unfolded protein response from the endoplasmic reticulum. $J$ Biol Chem 279: 25935-25938.

Zhang Z, Kobayashi S, Borczuk AC, Leidner RS, Laframboise T, Levine AD et al. (2010). Dual specificity phosphatase 6 (DUSP6) is an ETS-regulated negative feedback mediator of oncogenic ERK signaling in lung cancer cells. Carcinogenesis 31: 577-586.

Zhong Q, Gao W, Du F, Wang X. (2005). Mule/ARF-BP1, a BH3only E3 ubiquitin ligase, catalyzes the polyubiquitination of Mcl-1 and regulates apoptosis. Cell 121: 1085-1095.

Zhuang L, Lee CS, Scolyer RA, McCarthy SW, Zhang XD, Thompson JF et al. (2007). Mcl-1, Bcl-XL and Stat3 expression are associated with progression of melanoma whereas Bcl-2, AP-2 and MITF levels decrease during progression of melanoma. Mod Pathol 20: 416-426.

Zhuang L, Scolyer RA, Lee CS, McCarthy SW, Cooper WA, Zhang $\mathrm{XD}$ et al. (2009). Expression of glucose-regulated stress protein GRP78 is related to progression of melanoma. Histopathology 54: 462-470.

Zhuang L, Scolyer RA, Murali R, McCarthy SW, Zhang XD, Thompson JF et al. (2010). Lactate dehydrogenase 5 expression in melanoma increases with disease progression and is associated with expression of Bcl-XL and Mcl-1, but not Bcl-2 proteins. Mod Pathol 23: $45-53$.

This work is licensed under the Creative Commons
Attribution-NonCommercial-No Derivative
Works 3.0 Unported License. To view a copy of this license,
isit http://creativecommons.org/licenses/by-nc-nd/3.0/

Works 3.0 Unported License. To view a copy of this license, visit http://creativecommons.org/licenses/by-nc-nd/3.0/

Supplementary Information accompanies the paper on the Oncogene website (http://www.nature.com/onc) 